\title{
ASYMPTOTIC EQUIVALENCE OF VOLTERRA DIFFERENCE SYSTEMS
}

\author{
J. Morcha£o
}

\begin{abstract}
The purpose of this paper is to give some results on the asymptotic relationship between the solutions of a linear difference equation and its perturbed nonlinear equation.
\end{abstract}

\section{Introduction}

The problem of the asymptotic equivalence for systems of ordinary differential equations has been studied by many authors, as e.g. Brauer [3], Brauer and Wong [4], Boundorides and Georgiou [2], Lowell Lovelady [11], Morchało [13], Švec [17], Szufla [18], and others.

The problem of the asymptotic equivalence for integrodifferential equations has been studied by Morchało [14], Razapov [16], Talpalaru [19].

The problem of the asymptotic behavior of solutions of ordinary difference equations has been studied by Benzaid [1], Conffman [5], Drozdowicz, Popenda [6], Elaydi, Gyori [8], Li [10] and Pinto [15].

In this paper, we shall consider some results on the asymptotic relationship between the solutions of a linear Volterra difference equation and its perturbed nonlinear equation. The author knows only the works of Talpalaru [19], Ved and Gołovina [21], Ved and Kaptagaev [20], dealing with the above problem for special case.

\section{Notations and Definitions}

Here $N\left(n_{0}\right)=\left\{n_{0}, n_{0}+1, \ldots\right\}$, where $n_{0}$ is a natural number or zero; $R^{k}$ - the $k$-dimensional real euclidean space with the norm

$$
|x|=\sum_{i=1}^{k}\left|x_{i}\right|, \quad x=\left(x_{1}, \ldots, x_{k}\right)
$$


$M^{k}$ - the space of all $k \times k$ matrices $D=\left(d_{i j}\right)$ with the norm $|D|=$ $\max _{j} \sum_{i=1}^{k}\left|d_{i j}\right|, I$ - identity matrix.

We denote by $\Phi\left(N, R^{k}\right)$ the space of all functions from $N\left(n_{0}\right)$ into $R^{k}$. Let $\Phi_{1}=\Phi_{1}\left(N, R^{k}\right)$ be the Banach space in $\Phi$ of all bounded functions $u: N\left(n_{0}\right) \rightarrow R^{k}$ with norm $\|x\|=|x(n)|_{\Phi_{1}}=\sup \left\{|x(n)|: n \in N\left(n_{0}\right)\right\}$.

In this paper we consider the following systems of difference equations

$$
\begin{aligned}
& x(n+1)=[A+B(n)] x(n)+\sum_{r=0}^{n}[K(n-r)+Q(n, r)] x(r), \\
& x(n+1)=A x(n)+\sum_{r=0}^{n} K(n-r) x(r)+f(n)+F(n, x(n))
\end{aligned}
$$

thought et as perturbations of

$$
\begin{aligned}
& y(n+1)=A y(n)+\sum_{r=0}^{n} K(n-r) y(r), \\
& y(n+1)=A y(n)+\sum_{r=0}^{n} K(n-r) y(r)+f(n)
\end{aligned}
$$

where $x, y, f$ are $k$-dimensional vectors,

$$
\begin{aligned}
& A-\text { is a constant matrix } k \times k, \\
& B, K: N\left(n_{0}\right) \rightarrow M^{k} \\
& Q: N\left(n_{0}\right) \times N\left(n_{0}\right) \rightarrow M^{k}, \\
& F: N\left(n_{0}\right) \times U \rightarrow R^{k} \text { is for any } n \in N\left(n_{0}\right) \\
& \quad \text { continuous as a function of } x \in U \\
& \left(U-\text { a region in } R^{k}\right) .
\end{aligned}
$$

We define the resolvent matrix $R(n, m)$ of the equation

(2.3) $x(n+1)=[A+B(n)] x(n)+\sum_{r=0}^{n}[K(n-r)+Q(n, r)] x(r)+f(n)$

as the unique solution of the matrix difference equation [7]

$$
\begin{aligned}
R(n+1, m)=[A & +B(n)] R(n, m) \\
& +\sum_{r=m}^{n}[K(n-r)+Q(n, r)] R(r, m), \quad n \geq m,
\end{aligned}
$$


with $R(m, m)=I$.

Using the resolvent matrix $R(n, m)$ we can establish the following relation [7] (Variation of Constants Formula)

$$
x\left(n, 0, x_{0}\right)=R(n, 0) x_{0}+\sum_{r=0}^{n-1} R(n, r+1) f(r),
$$

where $x\left(n, 0, x_{0}\right)$ is the unique solution of the equation (2.3) satisfying $x\left(0,0, x_{0}\right)=x_{0}$.

Let $Y(n)$ denote the fundamental matrix of the system (2.1') [7]. Notice that $Y(0)=I$ and $y\left(n, 0, y_{0}\right)=Y(n) y_{0}$ is the unique solution of $\left(2.1^{\prime}\right)$ with $y\left(0,0, y_{0}\right)=y_{0}$.

Moreover,

$$
Y(n+1)=A Y(n)+\sum_{r=0}^{n} K(n-r) Y(r) .
$$

Remark [7]. We remark here that the resolvent matrix $R(n, m)$ for equations of nonconvolution type is closely related to the fundamental matrix $Y(n)$. By uniqueness of solutions, it is easy to see that for equations of convolutions type such as $\left(2.1^{\prime}\right), R(n, 0)=Y(n)$ and $R(n, m)=Y(n-m)$.

In this paper we consider the notion of asymptotic equivalence given by,

Definition. We say that the equations (2.1) and (2.1') or (2.2), (2.2') are asymptotically equivalent if, corresponding to each solution $x=x(n)$ of $(2.1),((2.2))$, there exists a solution $y=y(n)$ of $\left(2.1^{\prime}\right),\left(\left(2.2^{\prime}\right)\right)$ with the property

$$
\lim [x(n)-y(n)]=0 \text { as } n \rightarrow \infty \text { and conversely. }
$$

\section{Asymptotic equivalence}

We state the following lemma.

\section{Lemma 3.1. If}

1. $\varphi(n)$ is bounded on $N\left(n_{0}\right)$ and $\lim _{n \rightarrow \infty} \varphi(n)=\varphi(\infty)$ exists,

2. $\sum_{k=n_{0}}^{\infty}|g(k)|<\infty$ 
then

$$
\lim _{n \rightarrow \infty} \sum_{k=n_{0}}^{n} \varphi(n-k) g(k)=\varphi(\infty) \sum_{k=n_{0}}^{\infty} g(k)
$$

Theorem 3.2. Assume that

1. all solutions of the system (2.1') tend to finite limits as $n \rightarrow \infty$,

2. $\sum_{r=0}^{\infty}\left[|B(r)|+\sum_{s=0}^{r}|Q(r, s)|\right]<\infty$

3. $\operatorname{det} P \neq 0$, where $P=\lim Y(n)$ as $n \rightarrow \infty, P$ is a constant matrix,

4. $q=\sum_{r=0}^{\infty}\left[|B(r)||R(r, 0)|+\sum_{s=0}^{r}|Q(r, s)||R(s, 0)|\right]<1$.

Then,

a) corresponding to each solution $x=x(n) \in \Phi_{1}$ of (2.1), there exists a solution $y=y(n) \in \Phi_{1}$ of (2.1') such that (2.7) is satisfied provided that Conditions 1, 2 hold,

b) in Relation (2.7) the solution $y=y(n)$ of (2.1') is unique if Conditions 1, 2 and 3 are satisfied,

c) to each non-zero solution $x=x(n) \in \Phi_{1}$ of (2.1) there corresponds in Relation (2.7) a non-zero solution $y=y(n) \in \Phi_{1}$ of (2.1'), if Conditions 1, 2 and 4 hold and conversely,

d) in Relation (2.7) the solution $x=x(n)$ of (2.1) is unique if Conditions 1, 2, 3 and 4 are satisfied.

Proof: By Formula (2.5) the solutions $x(n)$ of (2.1) and $y(n)$ of $\left(2.1^{\prime}\right)$ can be written as

(3.1) $x(n)=Y(n) x_{0}+\sum_{r=0}^{n-1} Y(n-r-1)\left[B(r) x(r)+\sum_{s=0}^{r} Q(r, s) x(s)\right]$

and

$$
y(n)=Y(n) y_{0}, \quad n \in N
$$

Furthermore, from the Relations (3.1) and (3.2) we obtain

$$
\begin{aligned}
x(n)-y(n) & =Y(n)\left[x_{0}-y_{0}\right] \\
& +\sum_{r=0}^{n-1} Y(n-r-1)\left[B(r) x(r)+\sum_{s=0}^{r} Q(r, s) x(s)\right] .
\end{aligned}
$$


From Assumptions 1, 2 and (3.1) we obtain

$$
\begin{aligned}
& |x(n)| \leq|Y(n)|\left|x_{0}\right| \\
& \quad+\sum_{r=0}^{n-1}|Y(n-r-1)|\left[|B(r)||x(r)|+\sum_{s=0}^{r}|Q(r, s)||x(s)|\right] .
\end{aligned}
$$

Hence and difference inequality [9] we can easily obtain that all solutions of (2.1) are bounded.

Thus

$$
\sum_{n=0}^{\infty}\left|B(r) x(r)+\sum_{s=0}^{r} Q(r, s) x(s)\right|<\infty .
$$

By Assumption 1, Lemma 3.1 and Relations (3.3) (3.4) we get

$$
\begin{aligned}
\lim _{n \rightarrow \infty}[x(n) & -y(n)] \\
& =P\left\{x_{0}-y_{0}+\sum_{n=0}^{\infty}\left[B(n) x(n)+\sum_{s=0}^{n} Q(n, s) x(s)\right]\right\} .
\end{aligned}
$$

This shows that for arbitrary solutions $x(n)$ and $y(n)$ of $(2.1),\left(2.1^{\prime}\right)$ respectively Relation (2.7) hold iff

$$
P\left\{x_{0}-y_{0}+\sum_{n=0}^{\infty}\left[B(n) x(n)+\sum_{s=0}^{n} Q(n, s) x(s)\right]\right\}=0 .
$$

Equality (3.6) defines a relation between all solutions $x(n), y(n)$ of (2.1), (2.1'), respectively, for which (2.7) holds.

If $P=0$, then (3.6) means that (2.7) holds for arbitrary solutions $x(n), y(n)$ of $(2.1),\left(2.1^{\prime}\right)$ respectively.

On the other hand if $P \neq 0$, then for arbitrary solution $x(n)$ of $(2.1)$ we have

$$
y_{0}=x_{0}+\sum_{n=0}^{\infty}\left[B(n) x(n)+\sum_{s=0}^{n} Q(n, s) x(s)\right] \text {. }
$$

Hence for suitable solution $y(n)$ of (2.1') we conclude that (2.7) holds. Since Condition 3 holds, we claim that the solution $y(n)$ with the initial condition $y_{0}$ defined by (3.7) is unique in (2.7). 
From (2.5) for $f(n)=0$ and (3.7) we have

$$
y_{0}=x_{0}\left\{I+\sum_{n=0}^{\infty}\left[B(n) R(n, 0)+\sum_{s=0}^{n} Q(n, s) R(s, 0)\right]\right\}
$$

or

$$
\left(I+P_{0}\right) x_{0}=y_{0}
$$

where

$$
P_{0}=\sum_{n=0}^{\infty}\left[B(n) R(n, 0)+\sum_{s=0}^{n} Q(n, s) R(s, 0)\right] .
$$

Assume that $\left(I+P_{0}\right)^{-1}$ exists and $x(n) \neq 0$ for $n \in N\left(x_{0} \neq 0\right)$. Then, by (3.8), we have $y_{0} \neq 0(y(n) \neq 0$ for $n \in N)$. Such a matrix exists if, for example, $\left|P_{0}\right|<1[\mathbf{1 2}]$ (Banach Theorem's). From Assumption 4 it follows that $\left|P_{0}\right|<1$.

Let the initial condition $y_{0}$ of solution $y(n)=y\left(n, 0, y_{0}\right)$ be arbitrary.

From (3.8) we obtain

$$
x_{0}=\left(I+P_{0}\right)^{-1} y_{0}
$$

Hence for every solution $y(n) \neq 0$ on $N$ there exists a unique solution $x(n) \neq 0$ on $N$ such that $(2.7)$ holds and conversely.

Remark. If all solutions of (2.1') tends to zero as $n \rightarrow \infty$ and Condition 2 hold, then all solutions of (2.1) tend to zero as $n \rightarrow \infty$.

Now, we consider asymptotic equivalence between Equations (2.2) and $\left(2.2^{\prime}\right)$.

Lemma 3.3. Suppose that the following conditions hold:

1. every solution of (2.2') is bounded on $N$,

2. $\left|F\left(n, x_{1}\right)-F\left(n, x_{2}\right)\right| \leq g(n)|| x_{1}-x_{2} \mid$ for $n \in N, x_{1}, x_{2} \in U$,

3. $\sum_{n=0}^{\infty} g(n)<\infty$ and $\sum_{n=0}^{\infty}\left|F_{0}(n)\right|<\infty$ where $F_{0}(n) \equiv F(n, 0)$.

Then every solution of (2.2) is bounded on $N$ and

$$
|x(n)| \leq L M(n), \quad n \in N
$$


where

$$
\begin{aligned}
& Y_{0}=\sup _{N}|Y(n)| \\
& L=\sup _{N}\left|y_{0}(n)+\sum_{r=0}^{n-1} Y(n-r-1) F_{0}(r)\right|<\infty \\
& M(n)=\exp \left(Y_{0} \sum_{r=0}^{n-1} g(r)\right) \\
& y_{0}(n) \text { is a solution of (2.2'). }
\end{aligned}
$$

Proof: By the formula (2.5) the solution of (2.2) can be written as

$$
x\left(n, 0, x_{0}\right)=y_{0}(n)+\sum_{r=0}^{n-1} Y(n-r-1) F(r, x(r))
$$

where

$$
y_{0}(n)=Y(n) x_{0}+\sum_{r=0}^{n-1} Y(n-r-1) f(r), \quad n \in N .
$$

Furthermore, it follows from (2.5) and in view 1 that all solutions of (2.1') are bounded on $N$. Now, using the Relation (3.10) and the Condition 2, we get

$$
|x(n)| \leq L+Y_{0} \sum_{r=0}^{n-1} g(r)|x(r)|,
$$

which implies, by Gronwall inequality

$$
|x(n)| \leq L \exp Y_{0} \sum_{r=0}^{n-1} g(r)=L M(n) .
$$

Because the function $M_{1}(n)$ is bounded on $N$, we can conclude that the solution $x(n)$ of $(2.2)$ is also bounded on $N$.

Remark. From (3.9), we have

$$
|x(n)| \leq\left[Y_{0}\left|x_{0}\right|+f_{0}+Y_{0} F_{1}\right] M(n)
$$

where

$$
\begin{aligned}
& f_{0}=\sup _{N}\left|\sum_{r=0}^{n-1} Y(n-r-1) f(r)\right|<\infty, \\
& F_{1}=\sum_{n=0}^{\infty}\left|F_{0}(n)\right|<\infty
\end{aligned}
$$


Theorem 3.4. Let

1. all solutions of the system (2.1') tend to finite limits as $n \rightarrow \infty$,

2. Conditions 2 and 3 of lemma 3.3 hold,

3. $\sup _{n \in N}\left\{\sum_{r=0}^{n-1}\left|Z_{1}(n, r)\right||g(r)|+\sum_{r=n}^{\infty}\left|Z_{2}(n, r)\right||g(r)|<1\right\}$ where

$$
Z_{1}(n, r)=Y(n-r-1)-Y(n), \quad Z_{2}(n, r)=-Y(n) .
$$

Then for each solution $x(n)$ of (2.2) there corresponds a solution $y(n)$ of (2.2') such that (2.7) holds.

Moreover, suppose that Condition 3 of Theorem 3.2 holds. Then the solution $y(n)$ of (2.2') in Relation (2.7) is unique.

Let Conditions 1-3 hold and

4. $q_{1}=Y_{0} \sum_{n=0}^{\infty} g(n) M_{1}(n)<1$.

Then for each solution of (2.2) with $x_{0} \neq 0$ and

$$
\left|x_{0}\right|>\left(1-q_{1}\right)^{-1}\left[F_{2}+q_{1}\left(F_{1}+Y_{0}^{-1} f_{0}\right)\right]
$$

where

$$
F_{2}=\left|\sum_{n=0}^{\infty} F_{0}(n)\right|<\infty
$$

there corresponds a solution $y(n)$ of (2.2') with $y_{0} \neq 0$ such that (2.7) holds and conversely.

If, in addition the Condition 3 of Theorem 3.2 holds, then the solution $x(n)$ of (2.2) in Relation (2.7) is unique.

Proof: By 1 it follows that solutions of (2.2') are bounded on $N$. Then, the bounded properties of solutions of (2.2') imply that solutions of $\left(2.1^{\prime}\right)$ are bounded too. The first two parts of Theorem are easily verified (see Theorem 3.2 and Lemma 3.3). Since $P \neq 0$, then we can find a initial condition $y_{0}$ of the solution $y(n)$ of $\left(2.2^{\prime}\right)$ such that

$$
y_{0}=x_{0}+\sum_{n=0}^{\infty} F(n, x(n))
$$

where $x(n)$ is a given solution of $(2.2)$. 
Let $x_{0} \neq 0$, then from (3.13), (3.12) we have

$$
\begin{aligned}
\left|y_{0}\right| & \geq\left|x_{0}\right|-\left|\sum_{n=0}^{\infty} F(n, x(n))\right| \\
& \left.=\left|x_{0}\right|-\mid \sum_{n=0}^{\infty} F(n, x(n))-F(n, 0)\right]+\sum_{n=0}^{\infty} F(n, 0) \mid \\
& \geq\left|x_{0}\right|-\sum_{n=0}^{\infty}\left|F(n, x(n))-F_{0}(n)\right|-F_{2} \\
& \geq\left|x_{0}\right|-\sum_{n=0}^{\infty} g(n) M_{1}(n)\left[Y_{0}\left|x_{0}\right|+f_{0}+Y_{0} F_{1}\right]-F_{2} \\
& \geq\left|x_{0}\right|\left(1-q_{1}\right)-\left[\left(F_{1}+Y_{0}^{-1} f_{0}\right) q_{1}+F_{2}\right]>0 .
\end{aligned}
$$

Hence $y_{0} \neq 0$.

Let the initial condition $y_{0}$ of the solution $y(n)$ of (2.2') be arbitrary selection. Then by Conditions 1, 2 and (3.13) the solution $x(n)$ of $(2.2)$ be defined for all $n \in N$ and (2.7) be hold. By this means we give some conditions for existence and uniqueness of the solution $x(n)$ of $(2.2)$ in $\Phi_{1}$ which satisfied (3.13).

Since the equation (2.2) with initial condition $x_{0}$ is equivalent to the equation (3.10), then substituting for $x_{0}$ from (3.13) into (3.10)

$$
x(n)=y(n)+\sum_{r=0}^{n-1} Z_{1}(n, r) F(r, x(r))+\sum_{r=n}^{\infty} Z_{2}(n, r) F(r, x(r))
$$

where

$$
y(n)=Y(n) y_{0}+\sum_{r=0}^{n-1} Y(n-r-1) f(r)
$$

is arbitrary solution of $\left(2.2^{\prime}\right)$,

$$
\begin{aligned}
& Z_{1}(n, r)=Y(n-r-1)-Y(n) \\
& Z_{2}(n, r)=-Y(n) .
\end{aligned}
$$

Let $T$ be the operator defined for each $x \in \Phi_{1}$ by the equation

$$
T x(n)=\sum_{r=0}^{n-1} Z_{1}(n, r) F(r, x(r))+\sum_{r=0}^{\infty} Z_{2}(n, r) F(r, x(r)) .
$$


It is obvious that

$|F(n, x(n))| \leq|F(n, x(n))-F(n, 0)|+|F(n, 0)| \leq g(n)|x(n)|+|F(n, 0)|$.

The above inequality gives

$$
\begin{aligned}
|F x(n)| \leq \sum_{r=0}^{n-1}\left|Z_{1}(n, r)\right|[g(r) \mid & x(r)|+| F(r, 0) \mid] \\
& +\sum_{r=n}^{\infty}\left|Z_{2}(n, r)\right|[g(r)|x(r)|+|F(r, 0)|] .
\end{aligned}
$$

From Assumption 1, 2 we obtain $\|T x\|<\infty$. Thus $T$ maps $\Phi_{1}$ into itself. The operator $T$ is a contraction. In fact, let $x_{1}, x_{2} \in \Phi_{1}$,

$$
\begin{aligned}
\left|T x_{1}(n)-T x_{2}(n)\right| & \leq \sum_{r=0}^{n-1}\left|Z_{1}(n, r)\right|\left|F\left(r, x_{1}(r)\right)-F\left(r, x_{2}(r)\right)\right| \\
& +\sum_{r=n}^{\infty}\left|Z_{2}(n, r)\right|\left|F\left(r, x_{1}(r)\right)-F\left(r, x_{2}(r)\right)\right| \\
& \leq\left\|x_{1}-x_{2}\right\|\left\{\sum_{r=0}^{n-1}\left|Z_{1}(n, r)\right| g(r)+\sum_{r=n}^{\infty}\left|Z_{2}(n, r) g(r)\right|\right\} .
\end{aligned}
$$

This implies that $T$ is contraction in $\Phi_{1}$.

Remark. If all solutions of (2.2') tends to zero as $n \rightarrow \infty$ and the assumptions of Lemma 3.3 hold, then all solutions of (2.2) tend to zero as $n \rightarrow \infty$.

Remark. Let assumptions of theorem 3.4 hold and initial condition $x_{0}$ of the solution $x(n)$ of (2.2) implies

$$
\left|x_{0}\right|<\left(1+q_{1}\right)^{-1}\left[F_{2}-q_{1}\left(f_{0} Y^{-1}+F_{1}\right)\right]
$$

where

$$
F_{2}-q_{1}\left(f_{0} Y^{-1}+F_{1}\right)>0
$$

Then for each solution of $(2.2)$ with $x_{0} \neq 0$ corresponds solution $y(n)$ of $\left(2.2^{\prime}\right)$ with $y_{0} \neq 0$ such that $(2.7)$ holds and conversely. 


\section{References}

1. Z. Benzaid And D. A. Lutz, Asymptotic representation of solutions of perturbed systems of linear difference equations, Studies in Applied Mathematics 77 (1987), 195-221.

2. M. Boudorides AND D. Georgian, Asymptotic equivalence of differential equations with Stepanoff-bounded functional perturbation, Czech. Math. I. 32(107) (1982), 633-639.

3. F. Brauer, Nonlinear differential equations with forcing terms, Proc. Amer. Math. soc. 15 (1964), 758-765.

4. F. Brauer AND I. S. Wong, On the asymptotic relationships between solutions of two systems of ordinary differential equations I, Diff. Equations 6 (1969), 527-543.

5. C. V. Coffman, Asymptotic behavior of solutions of ordinary difference equations, Trans. Amer. Math. Soc. 110 (1964), 22-51.

6. A. Drozdowicz And J. Popenda, Asymptotic behavior of the solutions of second order difference equations, Proc. Amer. Math. Soc. 99 (1987), 135-140.

7. S. Elaydi, Periodicity and stability of linear Volterra difference system, J. Math. Anal. Appl. 182(2) (1994), 483-492.

8. S. Elaydi and I. Gyori, Asymptotic theory for delay and Volterra difference equations, J. Difference Equations and Applications 2 (1995) (to appear).

9. V. Lakshmikantham, S. Leela and A. A. Martynyuk, "Stability Analysis of Nonlinear Systems," Marcel Dekker, Inc., New York, 1989.

10. Z. H. LI, The asymptotic estimates of solutions of difference equations, J. Math. Anal. Appl. 94 (1983), 181-192.

11. D. Lowell Lovelady, Nonlinear Stepanoff-bounded perturbation problems, J. Math. Anal. Appl. 50 (1975), 350-360.

12. L. A. Lusternik And W. S. Sobolev, "Functional Analysis," Science Moskov, 1965 (russ.).

13. J. Morcha£o, Integral equivalence of two system of differential equations, Rendiconti Atti Accad. Naz. Lincei CCCLXXXII (1-2) (1985), 4-12.

14. J. Morcha£o, Asymptotic equivalence of Volterra integrodifferential equations, Radovi Matematički 7 (1991), 207-216.

15. M. Pinto, Discrete dichtomies, J. Comput. Math. Appl. 28(1-3) (1994), 2590-2700. 
16. G. RAzApov, Asymptotic properties of Volterra integrodifferential equations, Integrodifferential equations 4, Kirgiz (1967).

17. M. SvEC̆ , Asymptotic relationship between solutions of two systems of differential equations, Czech. Math. J. 24(99) (1974), 44-58.

18. S. Szufla, On the asymptotic relationshop between solutions of linear and quasilinear differential equations in Banach space, Commentations Math. XXVI (1986), 155-162.

19. P. TAlpalaru, Asymptotic behavior of perturbed difference equations, Atti Accad. Naz. Lincei LXIV(6) (1979), 563-571.

20. J. A. VeD And S. Kaptagaev, Asymptotic equivalence of difference equations, Integrodifferential equations 16, Kirgiz (1983), 181-196.

21. J. A. Ved And W. G. GoŁovina, Criteriums for the existence of constant solutions for the second order difference equations, Integrodifferential equations 16, Kirgiz (1983), 177-180.

\author{
Institute of Mathematics \\ Technical University of Poznan \\ 60-965 Poznan POLAND
}

Primera versió rebuda el 29 de Novembre de 1994, darrera versió rebuda el 31 de Març de 1995 